\title{
Contralateral Superior Cerebellar Artery Syndrome : A Consequence of Brain Herniation
}

\author{
Meysam Mohseni, M.D., 'Zohreh Habibi, M.D., ${ }^{2}$ Farideh Nejat, M.D. ${ }^{2}$ \\ Department of Neurosurgery, Imam Khomeini Hospital, Tehran University of Medical Sciences, Tehran, Iran \\ Department of Pediatric Neurosurgery, ${ }^{2}$ Children's Hospital Medical Center, Tehran University of Medical Sciences, Tehran, Iran
}

Vascular compromise is a well-known consequence of brain herniation syndromes. Transtentorial brain herniation most often involves posterior cerebral arteries. However, isolated involvement of contralateral superior cerebellar artery (SCA) during unilateral impending brain herniation is reported only once and we present another case of this exceedingly rare entity. A 24-year-old man was referred to us with impending herniation due to a multiloculated hydrocephalus, and during the course of illness, he developed an isolated SCA ischemia in the opposite side of the most dilated entrapped horn. In the current article we discuss the probable pathophysiologic mechanisms of this phenomenon, as well as recommending more inclusive brain studies in cases suspected of Kernohan-Woltman notch phenomenon in unilateral brain herniation. The rationale for this commentary is that contralateral SCA transient ischemia or infarct might be the underdiagnosed underlying pathomechanism of ipsilateral hemiparesis occurring in many cases of this somehow vague phenomenon.

Key Words : Superior cerebellar artery $\cdot$ Stroke $\cdot$ Cerebral hernia $\cdot$ Contralateral.

\section{INTRODUCTION}

The incidence of stroke rises exponentially with age and conversely is very low in young adults ${ }^{3)}$. The main challenge in the management of a young adult with acute stroke is the identification of potential cause. Although large atheroma, small-vessel disease, and atrial fibrillation have major roles in cases of stroke in elderly ${ }^{1,6)}$, these events are much less frequent in young adults. Several underlying cardiovascular, hematologic, metabolic, autoimmune and infectious diseases are proposed as the causes of juvenile onset ischemic stroke ${ }^{4)}$. More- over, there are numerous reports on intracranial vascular compromise as a consequence of brain herniation syndromes, most notably posterior cerebral artery (PCA) infarct following transtentorial herniation. However, there is only one report of unilateral brain herniation resulting in contralateral superior cerebellar artery (SCA) infarct in the literature ${ }^{10)}$.

Here, we present a young man in whom high intracranial pressure (ICP) and the resultant impending unilateral brain herniation led to contralateral SCA infarct. The probable underlying pathomechanisms are discussed.

- Received : February 26, 2014 •Revised : May 24, 2014 •Accepted : June 20, 2014

- Address for reprints : Zohreh Habibi, M.D.

Department of Pediatric Neurosurgery, Children's Hospital Medical Center, Tehran University of Medical Sciences, Tehran 1419733141, Iran Tel : +98-2166920983, Fax : +98-2122199377, E-mail : zohreh_h56@yahoo.com

This is an Open Access article distributed under the terms of the Creative Commons Attribution Non-Commercial License (http://creativecommons.org/licenses/by-nc/4.0) which permits unrestricted non-commercial use, distribution, and reproduction in any medium, provided the original work is properly cited. 


\section{CASE REPORT}

A 24-year-old man, fully awake and aware with no significant past medical history, admitted in neurosurgery ward due to a long-lasting history of diffuse constant non-throbbing headache and recent exacerbation accompanied with nausea and vomiting. His past medical history was unremarkable. In physical examination neither motor/sensory deficit nor cranial nerve dysfunction was detected, but bilateral small pale optic disks with sharp margins were evident in direct ophthalmoscopy. The patient underwent brain imaging to assess the potential causes of intracranial hypertension. Magnetic resonance imaging (MRI) revealed multiple septations in the right lateral ventricle resulting in a multiloculated appearance of the ventricle and biventricular hydrocephalus with the right side being apparently dominant (Fig. 1). Considering long history of headache, the septated hydrocephalus was assumed to be potentially caused by an old undiagnosed intraventricular event like hemorrhage or inflammation but remained compensated for a while. The patient was scheduled for an elective microsurgical fenestration of intraventricular septa to eliminate the intraventricular blocks and restore the normal cerebrospinal fluid (CSF) pathway. The procedure was performed via entering the right occipital horn where numerous arachnoid adhesions and bands were encountered which were released using a blunt hook. The most prominent adhesions
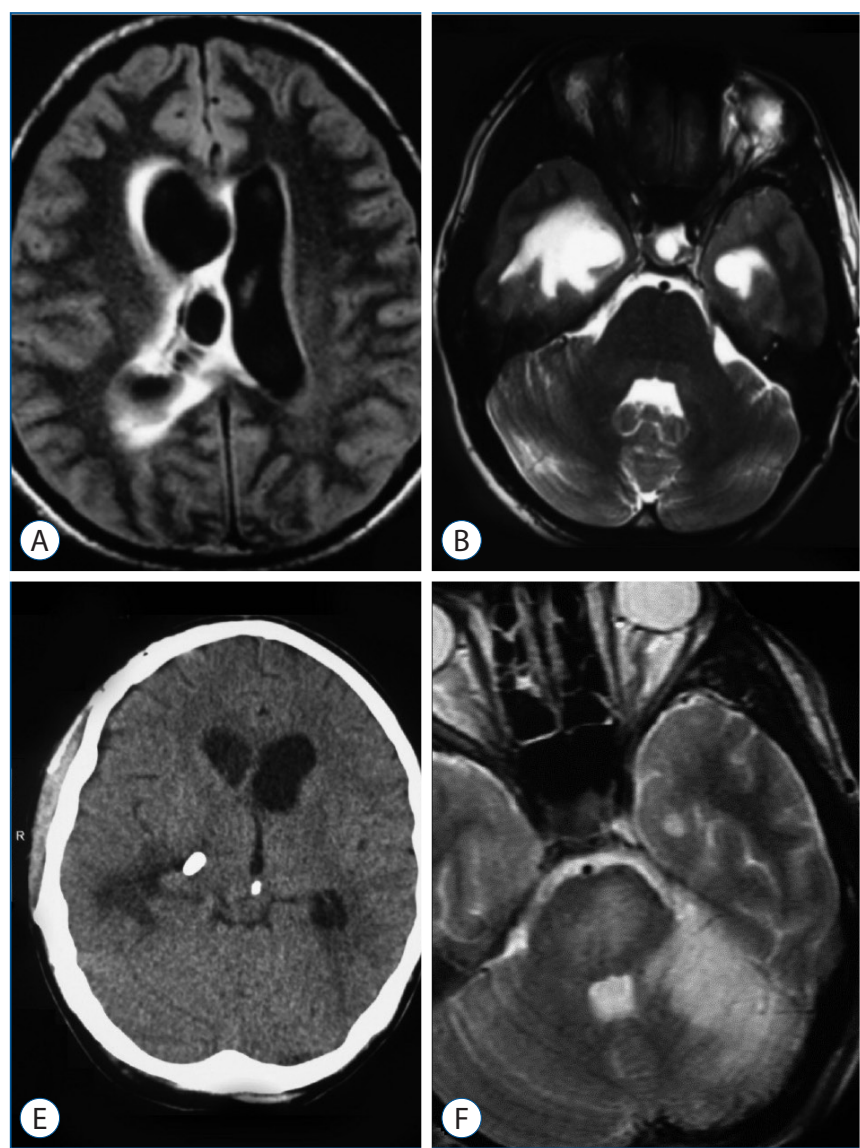
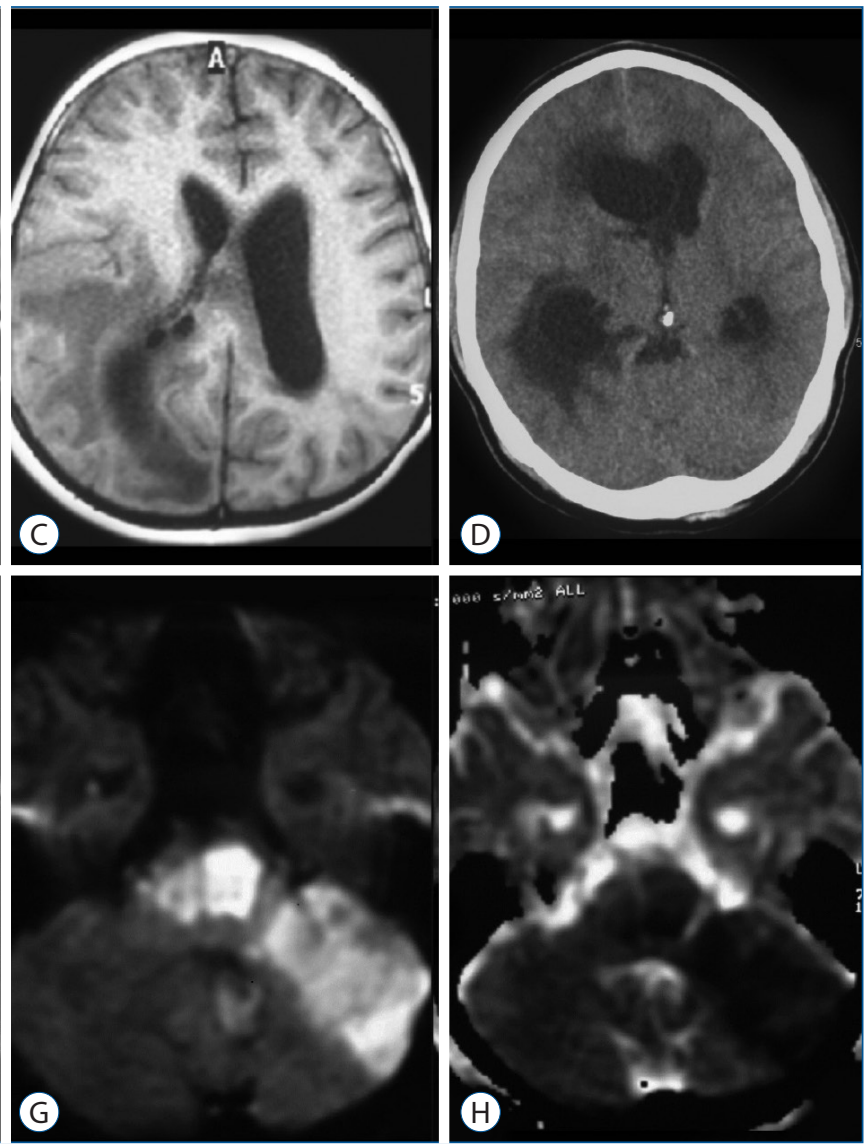

Fig. 1. A : Axial FLAIR brain MRI demonstrating multiple intraventricular septations causing biventricular hydrocephalus with periventricular edema on the right side and midline shift to the left. B : Infratentorial axial T2 weighted image showing normal left SCA territory at the first admission. C : Brain MRI after microsurgical fenestration showing relieved intraventricular loculation. D : Brain CT on readmission demonstrating severe hydrocephalus with trapped trigone and temporal horn of right lateral ventricle and midline shift to the opposite side. E : Brain CT after EVD procedure showing improved hydrocephalus and relieved sulcal effacement. F-H : Axial T2 MRI, diffusion weighted imaging, and apparent diffusion co-efficient map after patient's deterioration in the second admission demonstrating left SCA ischemic infarct. FLAIR : fluid-attenuated inversion recovery, MRI : magnetic resonance imagine, SCA : superior cerebellar artery, EVD : external ventricular drainage. 
were seen in the area of right trigone, creating multiple entrapped cavities whose walls were opened with sharp dissection. At the end of procedure, the ventricle seemed to be free of cavitation and the foramen of Monro was seen without previously adhesive barriers. The postoperative period was uneventful and the patient improved in terms of headache and vomiting. Postoperative images showed resolved hydrocephalus without formerly sulcal effacement or ventricular septation (Fig. 1). The patient discharged home on the third postoperative day with good general conditions and no new neurological deficits.

He readmitted to emergency ward 12 days after the first surgery while being confused (Glasgow coma scale : 13/15) for the last 2 days. A relatively rapid decline in the level of consciousness occurred within the first hours of admission and his right pupil's response to light became poor. Physical exam revealed repeated right hemiparesis. Considering the apparent unilaterally trapped right atrium and temporal horn on new images associated with significant periventricular edema and midline shift (Fig. 1), an emergent external ventricular catheter was inserted in the right lateral ventricle's trigone which resulted in rapid recovery of consciousness. During the procedure, CSF was found to have very high pressure, light pinkish color, and clear appearance. The analysis of CSF revealed 600 red blood cells, 0 white blood cells, and no bacteria in direct smear. After 48 hours with the patient being relatively well and the result of CSF culture being negative, ven- triculoperitoneal shunt was inserted in the trapped ventricular site. The rationale for early ventriculoperitoneal shunt procedure was to avoid probable external ventricular drainage infection, besides, there was no better option to manage trapped ventricle after a failed fenestration surgery. On postoperative images, hydrocephalus was resolved following shunting, but an acute ischemic stroke within the whole territory supplied by left SCA was evident that was not obvious in his first brain MRI study (Fig. 1). Brain CT- angiography was performed seven days after readmission which showed the left SCA being patent with no other vascular compromise or disease throughout the territory under study (Fig. 2).

Due to the odd location of the infarct, other probable causes of juvenile stroke were investigated. Trans-thoracic and transesophageal echocardiography precluded the probable cardioembolic causes or underlying cardiac anomalies such as endocarditis, valvular heart diseases and patent foramen ovale. Peripheral blood smear and hemoglobin electrophoresis and complete coagulation profile studies demonstrated no hematologic abnormalities. Comprehensive investigations for suspected autoimmune, metabolic, and infectious causes of juvenile stroke were unremarkable.

On the 9th day of shunting procedure the patient discharged with good general condition and full consciousness. Subsequently, hemiparesis subsided within about 6 weeks by the aid of physiotherapy.
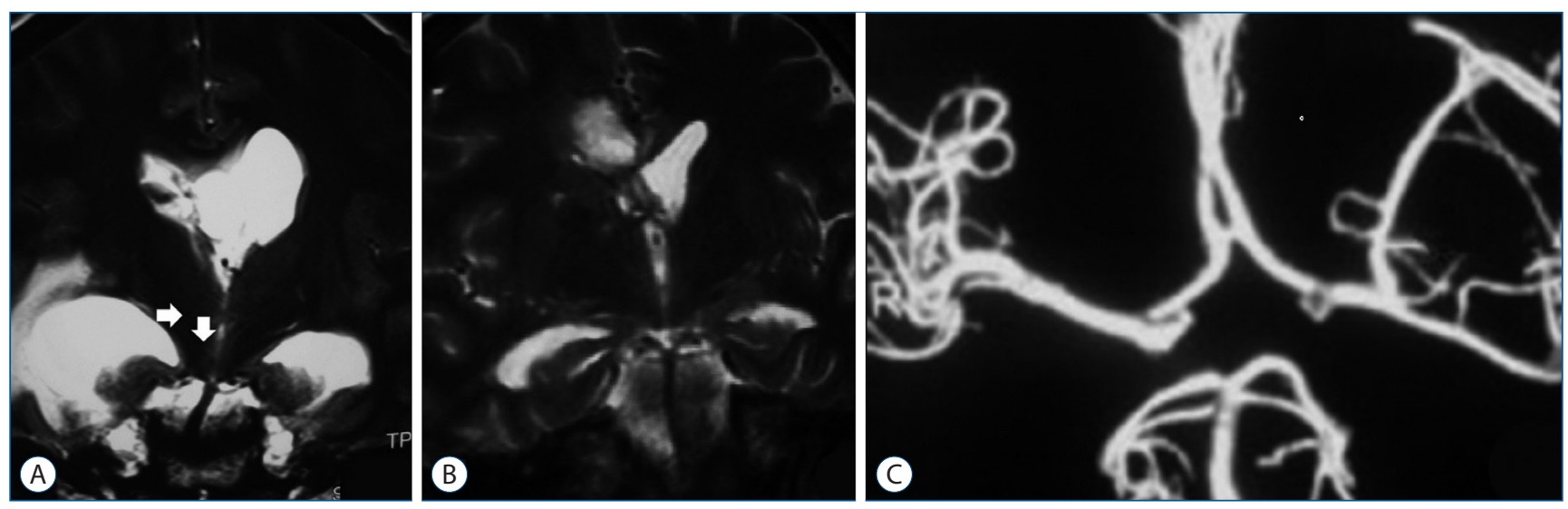

Fig. 2. A : Coronal T2 image in the phase of unilateral supratentorial hypertension showing upper basilar artery and its tributaries being distorted anatomically (arrows) potentially due to the right medial temporal lobe sliding down through the tentorial notch. B : Coronal T2 image following supratentorial decompression demonstrating the aforementioned structures regaining their normal anatomical relationships. C: Brain CT-angiography showing left SCA being patent with no other vascular compromise throughout the whole territory. SCA : superior cerebellar artery. 


\section{DISCUSSION}

Cerebral Infarction within the territory supplied by PCA as a consequence of transtentorial brain herniation has been well documented since several years ago ${ }^{2,7-9)}$. In contrast, only one case of SCA infarct complicating brain herniation has been defined in the literature, referring to a child with a brain tumor whose consciousness deteriorated due to ongoing brain herniation. In MRI taken 8 days later, ipsilateral PCA and surprisingly contralateral SCA infarct were evident ${ }^{10)}$.

Our patient is a further case who developed contralateral SCA infarct due to brain herniation. We assume the potential etiology to be vascular anatomy distortion in the tentorial incisura during herniation. Comparing the results of the postdecompression MR images with the MRI during hydrocephalic phase (demonstrating anatomical distortion and deviation of the basilar artery and both SCAs and PCAs), reestablishment of normal vascular anatomy in the tentorial incisura region after decompression is evident. Obviously this anatomic distortion should be much more severe during the acute phase of herniation when the patient has no time to undergo further and more precise cerebrovascular imaging studies (Fig. 2). Since the left SCA was patent in CT-angiographic study on the seventh day after supratentorial decompression and the basilar tip and its arterial branches regained their normal anatomic tension-free positions (Fig. 2), it seems that the pressure on the upper brainstem (by the right medial temporal lobe) and the consequent local vascular distortion remains the most probable cause of the vascular compromise and the resulting ischemic infarct. The infarction could be either due to contralateral SCA kinking or its origin being compressed between the upper basilar trunk and the tentorial incisural edge (particularly when a narrow tentorial incisura or a high riding basilar apex is present).

According to the present case, there are several points that merit attention : first, for developing vascular compromise, there is no need for the herniation process to be completed. This fact emphasizes on the short opening time that the raised intracranial pressure should be addressed in order to avoid its grave consequences. Second, contralateral SCA may possibly be more vulnerable than the ipsilateral one during unilateral brain herniation, albeit only two cases including this case are reported so far and the idea requires to be confirmed by the future similar reports. Third, SCA infarct during brain herniation syndromes might be much more frequent than what is being described as a rare event. This condition may be under-recognized due to its relatively silent presentation in an acutely ill patient or misdiagnosed as Kernohan-Woltman notch phenomenon (KWNP). KWNP is a well known syndrome since 1929 consisting of ipsilateral weakness presumably due to compression of contralateral crus cerebri to the tentorial incisura during impending or ongoing unilateral brain herniation $^{5}$. It is probable that contralateral SCA ischemia or infarct during an impending unilateral brain herniation is the missing pathophysiologic basis for some of cases diagnosed as KWNP. Accordingly, further evaluation of the patients with high ICP and ipsilateral hemiparesis using cerebrovascular imaging modalities (such as brain MR/CT-angiography or more precisely perfusion based images) may be required to investigate this hypothesis.

\section{CONCLUSION}

Contralateral SCA transient ischemia or infarct might be the underadiagnosed pathomechanism of ipsilateral hemiparesis in many cases diagnosed as KWNP.

\section{References}

1. Arnold M, Halpern M, Meier N, Fischer U, Haefeli T, Kappeler L, et al. : Age-dependent differences in demographics, risk factors, co-morbidity, etiology, management, and clinical outcome of acute ischemic stroke. J Neurol 255 : 1503-1507, 2008

2. Chungpieboonpatana A, Ratanalert S, Ladpli S: Occipital lobe infarction following descending transtentorial herniation. J Med Assoc Thai 71 : 553-560, 1988

3. Correia M, Silva MR, Matos I, Magalhães R, Lopes JC, Ferro JM, et al. : Prospective community-based study of stroke in Northern Portugal : incidence and case fatality in rural and urban populations. Stroke 35 : 2048-2053, 2004

4. Ferro JM, Massaro AR, Mas JL : Aetiological diagnosis of ischaemic stroke in young adults. Lancet Neurol 9 : 1085-1096, 2010

5. Kernohan JW, Woltman HW : Incisura of the crus due to contralateral brain tumor. Arch Neurol Psychiatry 21 : 274-287, 1929

6. Marini C, De Santis F, Sacco S, Russo T, Olivieri L, Totaro R, et al. : Contribution of atrial fibrillation to incidence and outcome of ischemic stroke : results from a population-based study. Stroke 36 : 1115-1119, 2005

7. McOmish D, Pike J : Occipital lobe infarction following descending transtentorial brain herniation. Australas Radiol 25 : 9-12, 1981 
8. Muthukumar N, Gurunathan J, Jawahar G, Gajendran R : Occipital lobe infarction caused by tentorial herniation. J Indian Med Assoc 90 : 161, 1992

9. Sato M, Tanaka S, Kohama A, Fujii C : Occipital lobe infarction caused by tentorial herniation. Neurosurgery $18: 300-305,1986$
10. Wang KC, Hong SK, Kim IO, Cho BK, Han DH : Infarction of the territory supplied by the contralateral superior cerebellar artery in a case of descending transtentorial herniation. Childs Nerv Syst 11 : 432-435, 1995 